\title{
A Rare Co-Occurrence of Triple Mutations in JAK2, CALR, and $M P L$ in the Same Patient with Myelofibrosis
}

\author{
Sherine J. Thomas $\mathbb{1 D}^{1}$ and D. P. Dash $\mathbb{D}^{2}$ \\ ${ }^{1}$ Hematology and Oncology, Northside Hospital Cancer Institute, Georgia Cancer Specialists, Atlanta, GA, USA \\ ${ }^{2}$ Molecular Oncology \& Genetics (MOGL), Versiti Wisconsin (Formerly Known as Blood Center Wisconsin), \\ Milwaukee, WI, USA \\ Correspondence should be addressed to Sherine J. Thomas; sherine.thomas@gacancer.com
}

Received 9 May 2021; Revised 8 November 2021; Accepted 21 December 2021; Published 21 February 2022

Academic Editor: Tatsuharu Ohno

Copyright (c) 2022 Sherine J. Thomas and D. P. Dash. This is an open access article distributed under the Creative Commons Attribution License, which permits unrestricted use, distribution, and reproduction in any medium, provided the original work is properly cited.

\begin{abstract}
Background. The diagnosis and prognostication of myeloproliferative neoplasm rely on the presence of driver mutations in JAK2, calreticulin (CALR), and MPL mutations. In the past, the presence of these mutations was thought to be mutually exclusive. Since then, there have been multiple reports of the presence of dual mutations. The presence of all three driver mutations in the same patient with myelofibrosis has not been previously described. Case. A 73-year-old female underwent a hematological workup in our facility after a routine hemogram performed prior to complex ophthalmological surgery revealed severe thrombocytosis. A comprehensive workup including an NGS panel for MPN driver mutations demonstrated that she had a calreticulin type-1 mutation, a JAK2 exon 14 (JAK2L611S) mutation, and an abnormal hotspot variant for MPL with VAF1\%. A bone marrow biopsy confirmed a myeloproliferative neoplasm with grade 2 reticulin fibrosis suggesting primary myelofibrosis. Molecular profiling of bone marrow confirmed the previously noted mutations and an MPLW515R mutation. The patient was started on treatment with hydroxyurea and aspirin with improvement in platelet count and resolution of anemia. Discussion. The clinical significance of the presence of multiple driver mutations in the same patient is not well understood at this time. There have been 11 publications between 2014 and 2020 that have described dual mutations of JAK2V617F, MPL, and CALR mutations. The JAK2 exon 14 mutation noted, in this case, is JAK2L611S which has not previously been reported in MPN and only reported in 5 cases in the COSMIC database. The JAK2 exon 14 mutation identified in this case is not an established driver mutation for myeloproliferative neoplasm, and its clinical implication remains unknown. Conclusions. The above case in addition to recent case reports and case series supports the use of broader NGS sequencing panels for diagnosis and prognostication of MPN. These mutations should not be considered mutually exclusive. The clinical behavior and prognosis of the subgroup with multiple mutations need to be studied in larger series.
\end{abstract}

\section{Introduction}

The molecular basis of myeloproliferative neoplasms (MPNs) came into the limelight in 2005 with the identification of JAK2 mutations in almost all cases of polycythemia vera and most essential thrombocythemia (ET) [1-4]. In 2006, somatic mutations in myeloproliferative leukemia virus oncogene (MPL) provided additional mechanistic understanding of the JAK-STAT pathway activation in MPN [5]. In 2013, somatic mutations in calreticulin $(C A L R)$ were reported as a driver mutation of essential thrombocythemia [6].
In clinical practice, these three driver mutations serve as diagnostic and prognostic markers during workup of myeloproliferative neoplasms. JAK2 mutations are present in most cases of polycythemia vera. Somatic mutation of codon 515 in exon 10 of the "myeloproliferative leukemia virus oncogene" $(M P L)$ is found in an estimated $3-4 \%$ of patients with essential thrombocythemia (ET) and 7\% of patients with primary myelofibrosis (PMF). Somatic mutations in calreticulin (CALR) are present in $25-30 \%$ of patients with essential thrombocytosis (ET) and primary myelofibrosis (PMF). CALR mutations have rarely been described in polycythemia vera. Importantly, mutations in CALR have 
previously been reported to be mutually exclusive with JAK2 or MPL mutations [6-8].

More recently, several case reports and case series have reported the coexistence of two driver mutations in Philadelphia chromosome-negative MPN, but these are known to be rare events. The presence of triple mutations in JAK2, $C A L R$, and $M P L$ in the same patient with MPN has not been previously reported, and therefore, here, we intend to present this unique case report.

\section{Case}

A 73-year-old African American female with a medical history of glaucoma and cataracts presented for hematology evaluation after routine hemogram performed before eye surgery demonstrated thrombocytosis and anemia. Her hemogram demonstrated hemoglobin of $9.9 \mathrm{~g} / \mathrm{dL}$ and hematocrit of $31.1 \%$. WBC count was normal at $9300 / \mathrm{mcL}$, absolute neutrophil count was $5952 \mathrm{mcg} / \mathrm{dL}$, basophils were $3 \%$ with absolute basophil count $279 / \mathrm{mcL}$, monocytes were $14 \%$, and absolute monocyte count was $1302 /$ microliter. Platelet count was elevated to $750,000 / \mathrm{mcL}$. She underwent workup for iron deficiency which showed a normal iron panel. Evaluation for myeloproliferative disorder (MPN) driver mutations was also performed.

The patient's peripheral blood sample was obtained for targeted gene sequencing. DNA extracted from peripheral blood was amplified via PCR with primers specific for gene targets-ABL1, ASXL1, BCOR, BRAF, CLAR, CDKN2A, CBL, CEBPA, CSF3R, DNMT3A, ETV6, EZH2, FLT3, GATA2, HRAS, IDH1, IDH2, FBXW7, IKZF1, JAK2, KIT, KRAS, MPL, MYD88, NF1, NPM1, NRAS, PHF6, PTEN, PTPN11, PRPF8, RB1, RUNX1, SETBP1, SF3B1, SH2B3, SRSF2, STAG2, TET2, TP53, UAF1, WT1, and ZRSR2. Specific genomic regions were targeted using a customized AmpliSeq Myeloid Next-Generation Sequencing (NGS) panel (Illumina), and sequencing by synthesis chemistry with paired-end 201 base pair read was performed. Bioinformatics sequence analysis pipeline was customized to limit analysis for only MPN gene targets.

Gene targets analyzed included all exons for $C A L R$ and selected exons with a high prevalence for the presence of pathogenic variants JAK2 (exons 12-15) and MPL (exons 3, 4,10 , and 12).

The following abnormal gene alterations were identified:

(1) CALR p.(Leu367ThrfsTer46) with a variant allele frequency of 46.5\% (c.1099_1150delCTTAAGGAG GAGGAAGACAAGAAACGCAAAGAGGAGGAG GAGGCAGAGG) (52 bp deletion, type 1).

(2) She was also found to have JAK2 p.(Leu611Ser) with variant allele frequency $23.2 \%($ c.1832T $>C)$.

(3) In addition to the above, abnormal testing for hotspot variant MPL p. Trp515Arg was detected with VAF $1 \%$, which is below the level of detection for that test (1.75\%): variant depth 56 and total depth 5554. Confirmatory testing with higher sensitive PCR-based locked nucleic acid (LNA) demonstrated the same MPL mutation (p.
Trp515Arg (W515R)) (tier I variant: NM_005373.2). The confirmatory testing for $M P L$ exon 10 mutation analysis is validated based on locked nucleic acid (LNA) methods for the MPL W515R codon which offers a higher sensitivity than traditional Sanger sequencing assays. Based on the LNA methods of detection, the higher sensitive MPL assay can detect mutation at the MPL W515R codon with low allele burden as low as $2 \%$ or below.

Given the unusual genetic mutation profile, a bone marrow biopsy was undertaken to quantify bone marrow fibrosis.

Flow cytometry on the bone marrow aspirate specimen failed to identify any unique cell populations of myeloid or lymphoid origin. A small population of cells identified by surface markers, CD13, CD22, low-density CD20 5, moderate CD33, bright CD38, moderate CD45, bright CD123, and negative for CD19, and by side scatter properties as basophils were noted to comprise $2 \%$ of the total nucleated sample. Monocytes were $6 \%$ of the nucleated sample, granulocytes $59 \%$, and lymphocytes $25 \%$.

Morphologically, the core biopsy demonstrated a hypercellular marrow (95\% cellularity) with trilineage hematopoiesis, myeloid-to-erythroid ratio $>5: 1$, with atypical megakaryocytic hyperplasia, moderately increased fibrosis, but no increase in blasts (Figures 1 and 2). Karyotype was noted to be normal-46, XX [20].

Based on the above workup, the patient was diagnosed with chronic myeloproliferative neoplasm-primary myelofibrosis.

The patient was started on treatment with hydroxyurea for cytoreduction and on aspirin for thromboprophylaxis, with improvement in the platelet count (Figure 3). She had improvement and normalization of hemoglobin (Figure 4).

Peak platelet count was $976,000 / \mathrm{mcL}$ at the time of initiation of cytoreductive therapy with hydroxyurea. Within 4 months of starting cytoreduction with hydroxyurea, the patient was noted to have normalization of the platelet count to $220,000 / \mathrm{mcL}$. WBC count and neutrophils remained stable, and anemia resolved. The patient remained stable and continued to tolerate hydroxyurea therapy. She has remained on aspirin for thromboprophylaxis.

\section{Discussion}

The presence of driver mutations has diagnostic, prognostic, and therapeutic implications in myeloproliferative disorders. To our knowledge, this is the first case of a patient with ET identified to have triple mutations JAK2 L611S, CALR type 1, and tier 1, MPL W515R, co-occurring in the same patient.

As mentioned above, the presence of multiple driver mutations in patients with MPN is well described, but they are rare events. For example, in a study evaluating the prevalence of MPN driver mutations in 928 Chinese patients, the JAK2 V617F and CALR exon 9 dual mutation was identified in six $(0.6 \%)$ patients, and the coexistence of JAK2 V617F and MPL exon 10 was even more uncommon, occurring in two (0.2\%) patients [9]. 


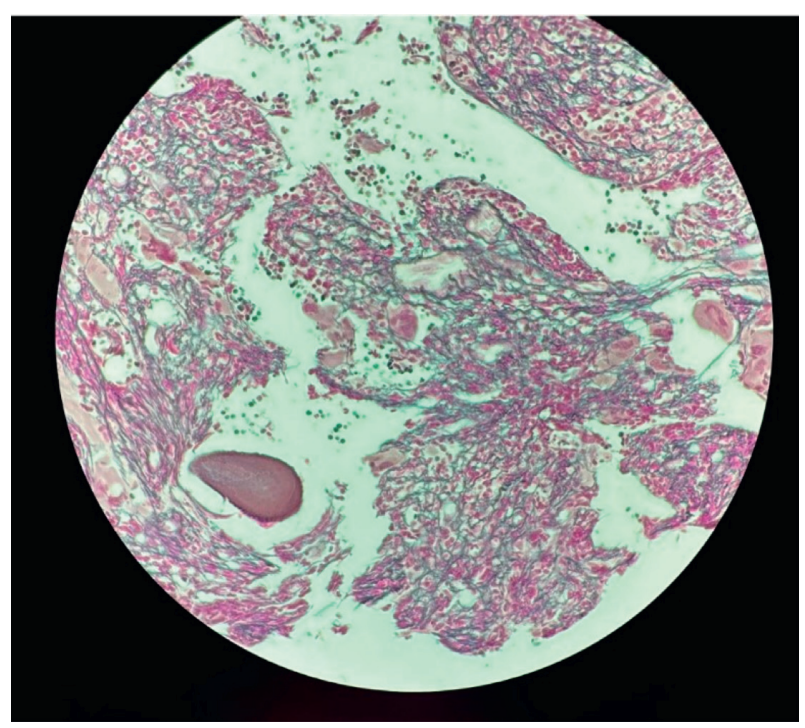

FIGURE 1: Reticulin-stained bone marrow core biopsy showing increased reticulin fibrosis. WHO* classification MF2 $\left({ }^{*}\right.$ World Health Organization).

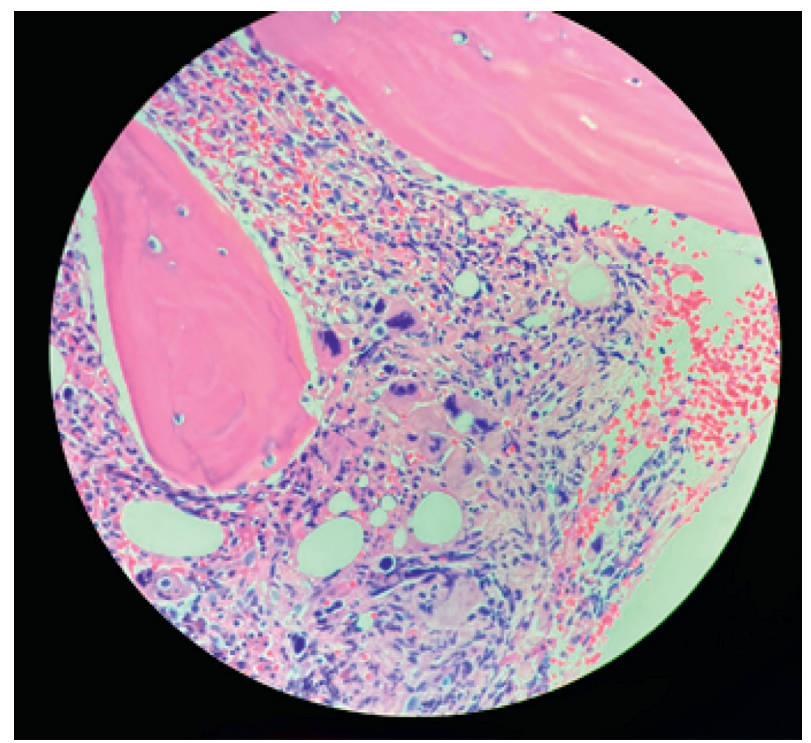

Figure 2: H\&E-stained section of bone marrow core biopsy showing atypical megakaryocytic hyperplasia.

JAK2 V617F mutation was the first and the most wellcharacterized driver mutation in myeloproliferative neoplasm. The patient described in this report had a less common JAK2 p.L611S mutation. We note that JAK2 p.L611S mutation is not a recognized driver mutation in MPN. However, given the clinical relevance of JAK2 mutations in MPN, we feel that this patient's less common JAK2 mutation profile should be described.

3.1. JAK2 p.L611S Mutation. Janus kinase 2 (JAK2) helps in modulating response to extracellular cytokine signalling. While JAK2 is a nonreceptor TKI, it needs a cognate receptor for response. Activated JAK2 signalling is necessary for normal hematopoiesis including erythropoiesis and thrombopoiesis. Once activated, JAK2 triggers recruitment and phosphorylation of downstream molecules such as STAT3/5 and MAP kinase which in turn allows the translocation of signalling molecules to the nucleus and thereby activates transcription of functional proteins.

$J A K 2$ p.L611S is a missense mutation in exon 14 of the $J A K 2$ gene in chromosome 9. It results in the replacement of amino acid leucine to serine at codon 611 (p.L611S). This variant represents a hotspot mutation within the JAK2-JH1 interface including one in the $\mathrm{JH} 2$ domain (L611S) and is supposed to destabilize JH2-JH1 interaction and enhance $J A K 2$ signalling.

JAK2 p.L611S has been reported in the COSMIC database (COSV67633764, legacy identifier: COSM21361) for a total of 4 cases including PV (with comutation in JAK2 V617F), AML in conjunction with Down syndrome myeloid disorder (DS-AMKL) (comutated with GATA and NIPBL and 7qdel, B-ALL, and squamous cell carcinoma (COSM21361, February 2021). JAK2 p.L611S has been shown to be associated with tumorigenesis in animal models [10]. Although JAK2 p.L611S mutation has not been reported with myelofibrosis or essential thrombocytosis, it has been reported in a child with congenital thrombocytosis.

3.2. Coexistence of Multiple Driver Mutations. Most cases of myeloproliferative neoplasm are known to harbour driver mutations JAK2, MPL, or CALR in a mutually exclusive manner [11]. However, there have been cases of rare patients with 2 coexisting mutations.

Co-occurrence of CALR and MPL has been reported in one patient from India [12]. Similarly, a case report from South Korea of $123 \mathrm{MPN}$ patients reported the presence of JAK2 and CALR mutations in 7 (4\%) patients [13]. Another case from Korea in 2021 reported a case of comutation of JAK2 V617F and MPL mutation [14].

A case series of $11 \mathrm{MPN}$ patients published in 2018 with multiple driver mutations (including $B C R-A B L$ ) had 8 patients positive for $B C R-A B L$ and $J A K 2$ V617F mutations, 1 patient positive for $B C R-A B L$ and $C A L R$ type 2 mutations, and 2 patients (referenced in Table 1) negative for Philadelphia chromosome, and one each among them had JAK2 $\mathrm{V} 617 \mathrm{~F}+M P L, J A K 2 \mathrm{~V} 617 \mathrm{~F}$, and CALR mutations [22].

The clinical behaviour of patients with these rare comutations is not well understood because of the rarity of these events. The limited case reports regarding these comutations have provided some insights.

A previous case series has suggested that the presence of $J A K 2$ and CALR did not appear to affect the prognosis or clinical features of ET, thereby suggesting that the disease phenotype is dictated primarily by the JAK2 mutation. They also noted that ET patients harboring mutations in the JAK2 gene had inferior progression-free survival (PFS) regardless of the presence of a mutation in CALR. They also suggested that response to therapy was superior in patients bearing $J A K 2$ mutations as compared to patients with CALR mutations [16]. 


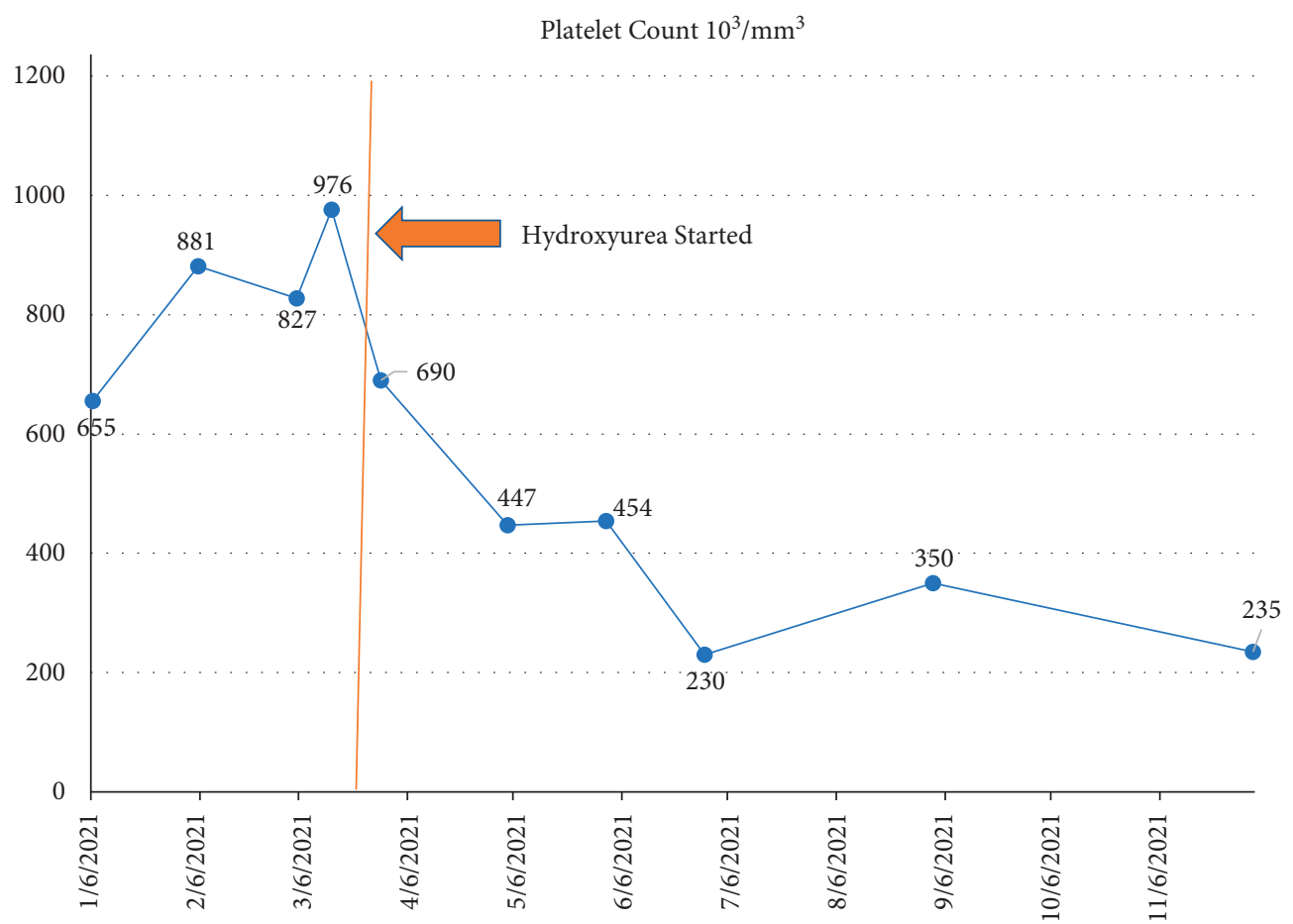

FIGURE 3: Graph of the platelet count at presentation and response to hydroxyurea.

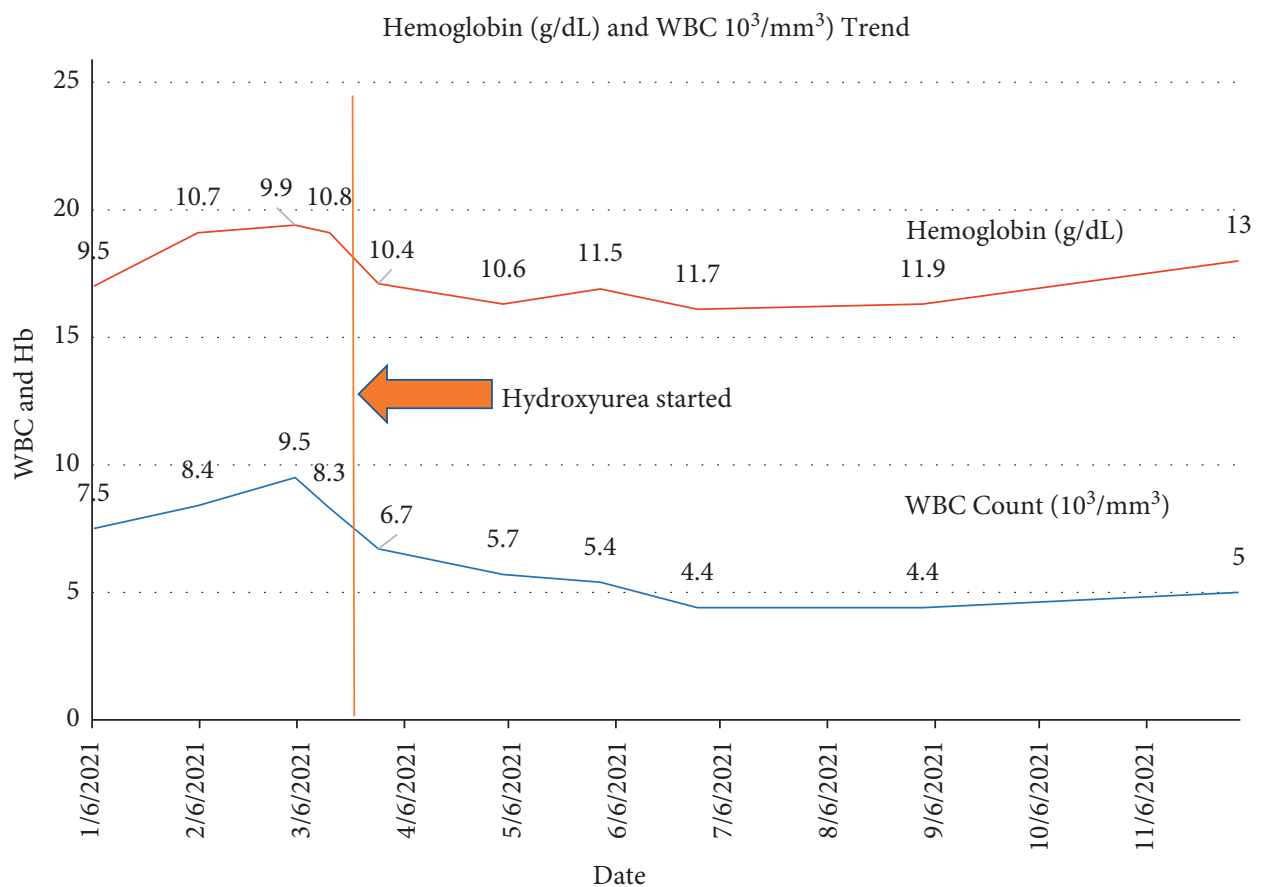

FIgURE 4: Graph of hemoglobin and white blood cell count trend and response to hydroxyurea.

Authors from a separate series suggested that the double mutated phenotype has a different clinical course compared to those with a single driver mutation [15].

Another larger case series of 40 double mutated patients with essential thrombocythemia showed that these patients with multiple mutations have a male predominance, the advanced age of onset, lower hemoglobin, and higher platelet count compared to the patients with a single mutation. However, the incidence of thrombosis was not different compared to patients with a single driver mutation [23].

The JAK2 p.L611S mutation identified in this case has not been recognized as a driver mutation of myeloproliferative neoplasm. However, given the seminal role of JAK2 
TABLE 1: A summary of case reports and case series with multiple driver mutations present in patients with ET and PMF.

\begin{tabular}{|c|c|c|c|c|c|c|c|c|}
\hline No. & Author & $N$ & MPN & JAK2 exon 14 & JAK2 exon 12 & MPL & CALR & Year \\
\hline 1 & McGaffin et al. [15] & 1 & - & V617F & - & - & Type 6 (48 bp del) & 2014 \\
\hline 2 & Kang et al. [16] & 7 & ET & V617F & - & - & All types $(1,2$, and 3$)$ & 2016 \\
\hline 3 & Rashid et al. [17] & 1 & ET & V617F & - & - & Type 1 (52 bp del) & 2016 \\
\hline \multirow[t]{2}{*}{4} & Cleyrat et al. [18] & 1 & ET & - & - & p.W515R & Type 1 (52 bp del) & 2017 \\
\hline & & 12 & - & V617F & - & Type not reported & - & 2017 \\
\hline \multirow[t]{3}{*}{5} & Jeromin et al. [19] & 6 & - & V617F & - & - & Type unreported & \\
\hline & & 1 & - & - & - & Type not reported & Type unreported & \\
\hline & & 3 & ET & V617F & - & W515L & - & 2017 \\
\hline \multirow[t]{2}{*}{6} & Usseglio et al. [20] & 1 & ET & V617F & - & W515R & - & \\
\hline & & 4 & ET & V617F & - & - & Type 1 (52 bp del) & \\
\hline 7 & Boddu et al. [21] & 1 & ET & $\begin{array}{c}\text { V617F (VAV } \\
<1 \%)\end{array}$ & - & - & Type 1 (52 bp del) & \\
\hline \multirow{2}{*}{8} & \multirow{2}{*}{ De Roeck et al. [22] } & 1 & ET & V617F & - & p.W515R & - & 2018 \\
\hline & & 1 & PMF & V617F & - & - & Type 1 (52 bp del) & \\
\hline \multirow{5}{*}{9} & \multirow{5}{*}{ Mansier et al. [23] } & 5 & - & V617F (VAF <5\%) & - & - & Type unreported & 2018 \\
\hline & & 32 & - & V617F & - & - & Type unreported & \\
\hline & & 11 & - & V617F & - & Type not reported & - & \\
\hline & & 2 & - & - & - & Type not reported & Type unreported & \\
\hline & & 1 & - & V617F & $\begin{array}{l}\text { Type not } \\
\text { reported }\end{array}$ & - & 2 CALR mutations & \\
\hline 10 & Ramanan et al. [12] & 1 & - & - & - & $\begin{array}{c}\text { p.W515R (VAF } \\
9.8 \%)\end{array}$ & $\begin{array}{c}\text { Type } 152 \text { bp deletion (VAF } \\
13 \% \text { ) }\end{array}$ & 2019 \\
\hline 11 & Zhou et al. [13] & 1 & PMF & - & - & p.X636W & CALR-p.364fs & 2020 \\
\hline
\end{tabular}

Table 1 is modified from Table 1 of Ramanan et al. [12].

mutations in these diseases, this patient's mutation profile is of interest.

\section{Conclusion}

To the best of our knowledge, this represents the first reported case of the coexistence of JAK2 p.L611S, CALR type 1, and tier $1 M P L$ mutation in the same patient with essential thrombocythemia. It is also the first reported case of JAK2 p.L611S in an adult with myelofibrosis. This case report highlights the increased recognition of cases with multiple mutations in JAK2, MPL, and CALR. Longer-term follow-up of patients with these comutations will lead to a greater understanding of their clinical behaviour and prognosis.

\section{Data Availability}

The data used to support the findings of this study, including high-resolution microscopic images and molecular test results, are included within the article.

\section{Conflicts of Interest}

The authors declare that they have no conflicts of interest.

\section{References}

[1] C. James, V. Ugo, J. -P. Le Couédic et al., "A unique clonal JAK2 mutation leading to constitutive signalling causes polycythaemia vera," Nature. vol. 434, no. 7037, pp. 1144-1148, 2005.

[2] R. Kralovics, F. Passamonti, A. S. Buser et al., "A gain-offunction mutation of JAK2 in myeloproliferative disorders,"
New England Journal of Medicine, vol. 352, pp. 1779-1790, 2005.

[3] R. L. Levine, M. Wadleigh, J. Cools et al., "Activating mutation in the tyrosine kinase JAK2 in polycythemia vera, essential thrombocythemia, and myeloid metaplasia with myelofibrosis," Cancer Cell, vol. 7, pp. 387-397, 2005.

[4] E. J. Baxter, L. M. Scott, P. J. Campbell et al., "Acquired mutation of the tyrosine kinase JAK2 in human myeloproliferative disorders," Lancet, vol. 365, pp. 1054-1061, 2005.

[5] Y. Pikman, B. H. Lee, T. Mercher et al., "MPL W515L is a novel somatic activating mutation in myelofibrosis with myeloid metaplasia," PLoS Medicine, vol. 3, p. e270, 2006.

[6] T. Klampfl, H. Gisslinger, A. S. Harutyunyan et al., "Somatic mutations of calreticulin in myeloproliferative neoplasms," New England Journal of Medicine, vol. 369, no. 25, pp. 2379-2390, 2013.

[7] J. Nangalia, C. E. Massie, E. J. Baxter et al., "Somatic CALR mutations in myeloproliferative neoplasms with nonmutated JAK2," New England Journal of Medicine, vol. 369, no. 25, pp. 2391-2405, 2013.

[8] E. Rumi, D. Pietra, V. Ferretti et al., "JAK2 or CALR mutation status defines subtypes of essential thrombocythemia with substantially different clinical course and outcomes," Blood, vol. 123, no. 10, pp. 1544-1551, 2014.

[9] Y. Lin, E. Liu, Q. Sun et al., "The Prevalence of JAK2, MPL, and CALR Mutations in Chinese Patients With BCRABL1-Negative Myeloproliferative Neoplasms," American Journal of Clinical Pathology, vol. 144, no. 1, pp. 165-171, 2015.

[10] M. Funakoshi-Tago, K. Tago, K. Sumi et al., "The acute lymphoblastic leukemia-associated JAK2 L611S mutant induces tumorigenesis in nude mice," Journal of Biological Chemistry, vol. 284, no. 19, pp. 12680-12690, 2009.

[11] T. Ayalew and T. Barbui, "Polycythemia Vera and essential thrombocythemia: 2021 update on diagnosis, risk- 
stratification, and management," American Journal of Hematology, vol. 95, no. 12, pp. 1599-1613, Article ID 3297493920, 2020.

[12] V. Ramanan, K. Kelkar, S. Ranade et al., "The clinical utility of a custom-developed targeted next-generation sequencing assay for detection of mutations associated with Philadelphia negative journal of hematopathology (2019) 12:163-168-167 chronic myeloproliferative neoplasms: two case examples with CALR exon 9 mutations," The International Journal of Molecular and Immuno Oncology, vol. 1, pp. 28-34, 2016.

[13] F. P. Zhou, C. C. Wang, H. P. Du, S. B. Cao, and J. Zhang, "Primary myelofibrosis with concurrent CALR and MPL mutations: a case report," World Journal of Clinical Cases, vol. 8, no. 22, pp. 5618-5624, 2020.

[14] M. Jang, M. Y. Seo, K. J. Choi, and D. -S. Hong, "A rare case of ET with coexisting JAK2 and MPL driver mutations," Journal of Korean Medical Science, vol. 35, 2020.

[15] G. McGaffin, K. harper, D. Stirling, and L. McLintock, "JAK2 V617F and CALR mutations are not mutually exclusive, findings from retrospective analysis of a small patient cohort," British Journal of Haematology, vol. 167, pp. 276-278, 2014.

[16] M. G. Kang, H. -W. Choi, J. H. Lee et al., "Co-existence of JAK2 and CALR mutations and their clinical implications in patients with ET.2016 Oncotarget, vol.7, no.35 57036-57049 Jang M.-A., M. Y. Seo, K. J. Choi and D. -S. Hong a rare case of ET with co-existing JAK2 and MPL driver mutations," Journal of Korean Medical Science, vol. 35, no. 23, 2020.

[17] M. Rashid, Z. Ahmed, S. Ahmed, M. Nadeem, N. Ahmed, and T. S. Shams, "Coexisting JAK2 V617F and CALR exon 9 mutation in essential thrombocythaemia," Indian Journal of Hematology and Blood Transfusion, vol. 32, pp. 112-116, 2016.

[18] C. Cleyrat, D. S. Chabot-Richards, D. T. Lynch et al., "Leukemic transformation of post-essential thrombocythemia myelofibrosis: a unique case presenting with double MPL and CALR mutations," Blood, vol. 130, no. 1, p. 4215, 2017.

[19] S. Jeromin, M. Meggendorfer, and A. Fasan, "Frequency of concurrent BCR-ABL1, JAK2, CALR and MPL mutations in a cohort of 5,545 cases with suspected MPN by a deep sequencing approach," EHA Learn Center, vol. 2017, Article ID 181088, 2017.

[20] F. Usseglio, N. Beaufils, A. Calleja, S. Raynaud, and J. Gabert, "Detection of CALR and MPL mutations in low allelic burden JAK2 V617F essential thrombocythaemia," Journal of Molecular Diagnostics, vol. 91, pp. 92-98, 2017.

[21] P. Boddu, D. Chihara, L. Masarova, N. Pemmaraju, K. P. Patel, and S. Verstovsek, "The co-occurrence of driver mutations in chronic myeloproliferative neoplasms," Annals of Hematology, vol. 97, pp. 2071-2080, 2018.

[22] L. De Roeck, L. Michaux, K. Debackere, E. Lierman, P. Vandenberghe, and T. Devos, "Coexisting driver mutations in MPN: clinical and molecular characteristics of a series of 11 patients," Hematology, vol. 23, pp. 785-792, 2018.

[23] O. Mansier, D. L. Paz, J. C. Lanotto et al., "Clinical and biological characterization of MPN patients harboring two driver mutations, a French intergroup of myeloproliferative neoplasms (FIM) study," American Journal of Hematology, vol. 93, pp. E84-E109, 2018. 